\title{
Article \\ Inhibition of the PI3K/mTOR Pathway in Breast Cancer to Enhance Response to Immune Checkpoint Inhibitors in Breast Cancer
}

\author{
Chi Yan ${ }^{1,2,+} \mathbb{D}$, Jinming Yang ${ }^{1,2,+}$, Nabil Saleh ${ }^{1,2} \mathbb{D}$, Sheau-Chiann Chen ${ }^{3}$, Gregory D. Ayers ${ }^{3}$, \\ Vandana G. Abramson ${ }^{4}\left(\mathbb{D}\right.$, Ingrid A. Mayer ${ }^{4}$ and Ann Richmond 1,2,*(D)
}

check for updates

Citation: Yan, C.; Yang, J.; Saleh, N.; Chen, S.-C.; Ayers, G.D.; Abramson, V.G.; Mayer, I.A.; Richmond, A. Inhibition of the PI3K/mTOR Pathway in Breast Cancer to Enhance Response to Immune Checkpoint Inhibitors in Breast Cancer. Int. J. Mol. Sci. 2021, 22, 5207. https://doi.org/ $10.3390 /$ ijms 22105207

Academic Editor: Alvaro Teijeira

Received: 20 April 2021

Accepted: 11 May 2021

Published: 14 May 2021

Publisher's Note: MDPI stays neutral with regard to jurisdictional claims in published maps and institutional affiliations.

Copyright: (c) 2021 by the authors. Licensee MDPI, Basel, Switzerland. This article is an open access article distributed under the terms and conditions of the Creative Commons Attribution (CC BY) license (https:// creativecommons.org/licenses/by/ $4.0 /)$.
1 Department of Veterans Affairs, Tennessee Valley Healthcare System, Nashville, TN 37212, USA; chi.yan@vanderbilt.edu (C.Y.); jinming.yang@vanderbilt.edu (J.Y.); nabil.saleh@vanderbilt.edu (N.S.)

2 Department of Pharmacology, Vanderbilt University School of Medicine, Nashville, TN 37240, USA

3 Department of Biostatistics, Vanderbilt University Medical Center, Nashville, TN 37232, USA; sheau-chiann.chen.1@vumc.org (S.-C.C.); dan.ayers@vumc.org (G.D.A.)

4 Department of Medicine, Vanderbilt University Medical Center, Nashville, TN 37232, USA; vandana.abramson@vumc.org (V.G.A.); ingrid.mayer@vumc.org (I.A.M.)

* Correspondence: ann.richmond@vanderbilt.edu; Tel.: +1-615-343-7777

+ These authors contributed equally to this work.

\begin{abstract}
Objectives: Inhibition of the PI3K/mTOR pathway suppresses breast cancer (BC) growth, enhances anti-tumor immune responses, and works synergistically with immune checkpoint inhibitors (ICI). The objective here was to identify a subclass of PI3K inhibitors that, when combined with paclitaxel, is effective in enhancing response to ICI. Methods: C57BL/6 mice were orthotopically implanted with syngeneic luminal/triple-negative-like PyMT cells exhibiting high endogenous PI3K activity. Tumor growth in response to treatment with anti-PD-1 + anti-CTLA-4 (ICI), paclitaxel (PTX), and either the PI3K $\alpha$-specific inhibitor alpelisib, the pan-PI3K inhibitor copanlisib, or the broad spectrum PI3K/mTOR inhibitor gedatolisib was evaluated in reference to monotherapy or combinations of these therapies. Effects of these therapeutics on intratumoral immune populations were determined by multicolor FACS. Results: Treatment with alpelisib + PTX inhibited PyMT tumor growth and increased tumor-infiltrating granulocytes but did not significantly affect the number of tumor-infiltrating $\mathrm{CD}^{+} \mathrm{T}$ cells and did not synergize with ICI. Copanlisib + PTX + ICI significantly inhibited PyMT growth and increased activation of intratumoral CD8 ${ }^{+} \mathrm{T}$ cells as compared to ICI alone, yet did not inhibit tumor growth more than ICI alone. In contrast, gedatolisib + ICI resulted in significantly greater inhibition of tumor growth compared to ICI alone and induced durable dendritic-cell, CD8 ${ }^{+}$T-cell, and NK-cell responses. Adding PTX to this regimen yielded complete regression in $60 \%$ of tumors. Conclusion: PI3K/mTOR inhibition plus PTX heightens response to ICI and may provide a viable therapeutic approach for treatment of metastatic BC.
\end{abstract}

Keywords: PI3K/mTOR; breast cancer; immune checkpoint inhibitor; cytotoxic T cells

\section{Introduction}

The phosphatidylinositol 3-kinase/protein kinase B/mammalian target of rapamycin (PI3K/mTOR) pathway is frequently altered in breast cancer (BC) [1]. The oncogenic activation of $\mathrm{PI} 3 \mathrm{~K} / \mathrm{mTOR}$ may occur through the mutation or overexpression of upstream regulators (e.g., epidermal growth factor receptor [EGFR, 4\%]; NRAS, 3\%; KRAS, 4\%), amplification or activating mutations in the PI3K catalytic subunit $\alpha$ (PI3KCA) $(47 \%)$, loss of function or expression of phosphatase and tensin homolog (PTEN) (6\%), or amplification of AKT (also known as protein kinase B, 5\%) (cBio-TCGA). PI3Ks are heterodimers and exist in four isoforms, namely $\mathrm{PI} 3 \mathrm{~K} \alpha, \beta, \gamma, \delta$. These isoforms convert phosphatidylinositol4,5-bisphosphate (PIP2) into phosphatidylinositol-3,4,5-trisphosphate (PIP3), which in turn controls a range of cellular actions including cell growth, migration, metabolism, survival, 
and proliferation [2]. PI3K $\alpha \beta$ are expressed ubiquitously and are often constitutively active in $\mathrm{BC}$, while $\mathrm{PI} 3 \mathrm{~K} \gamma \delta$ are predominately expressed in leukocytes, suggesting that, in addition to reprogramming tumor immune microenvironment, targeting PI3Ks will also have direct effect on breast cancer cells. However, extensive grade 3 and 4 toxicity present a major limitation of continuous inhibition of PI3Ks in patients [3], and new therapeutic combinations for PI3K inhibition in BC are needed.

The discovery of immune checkpoint inhibitors (ICIs) targeting cell death protein (PD)-1/PD-Ligand 1 (PD-L1) and cytotoxic T-lymphocyte-associated protein (CTLA)-4 is revolutionizing cancer treatments [4]. ICIs unleash anti-tumor immune responses, including $\mathrm{CD}^{+}$and $\mathrm{CD}^{+} \mathrm{T}$ cell, dendritic cell (DC), and macrophage effector function through blocking negative immune checkpoint proteins $[4,5]$. However, breast tumors are often unable to respond to ICIs because they are "immunologically cold", in part due to a relatively low mutational load, and many of these tumors feature an immunesuppressive tumor microenvironment (TME) [6]. Nevertheless, combining ICI therapy with the microtubule poison paclitaxel (PTX) induces immunogenic tumor cell death and enhances anti-cancer immunity [7]. Recently, nanoparticle albumin-bound (nab)-PTX combined with anti-PD-L1 antibody atezolizumab was FDA approved for treatment of locally advanced, unresectable TNBC based on the finding of when patients with PD-L1positive tumors were treated with this combination of therapy, there was an improvement in overall survival [8]. Furthermore, pre-clinical studies using combined treatment with PTX and either a $\beta$-isoform-sparing PI3K inhibitor taselisib or pan-AKT inhibitor ipatasertib reduced proliferative and metastatic effects of breast cancer cells, as compared to either therapeutic strategy alone [9]. We have shown in mouse models that BKM120, a pan-PI3K inhibitor, slows BC tumor growth and reduces metastasis, enhances antitumor immunity, and increases sensitivity to ICI [10]. However, severe toxicity of BKM120 was observed in patients due to the ability of BKM120 to cross the blood-brain barrier and induce severe depression, which resulted in withdrawal of the drug [11]. A phase II clinical trial study of combining PTX with mTOR inhibitor everolimus showed that while PTX is well tolerated, the combination treatment was associated with more adverse events without improvement in pathologic complete response or clinical response in patients with stage II/III triplenegative breast cancer (TNBC) [12]. Subsequently, clinical trials turned to examine effects of either isoform-specific or dual PI3K/mTOR inhibitors in patients with advanced or metastatic BC. To date, results from trials combining PI3K $\alpha$ or PI3K/mTOR inhibition with ICI therapy have not been reported. The objective of this study was to evaluate the effects of isotype-specific PI3K $\alpha$ inhibitors versus alternative pan-PI3K inhibitors or PI3K/mTOR pathway inhibitors in combination with PTX and ICI for treatment of BC.

Herein, we show that combined treatment with a PI3K $\alpha$ isoform-specific inhibitor, alpelisib, and PTX induces beneficial, but not durable, anti-tumor granulocyte responses in BC patient peripheral blood. Using the polyomavirus middle T (PyMT) murine breast cancer model, we validated the alpelisib induced anti-tumor granulocyte responses. However, the addition of ICI to this therapeutic regimen did not enhance response to alpelisib + PTX therapy. The pan-PI3K inhibitor, copanlisib, combined with ICI resulted in partial remission of PyMT breast tumor growth, which was associated with an elevation of $\mathrm{CD} 69^{+} \mathrm{CD} 8^{+}$ activated cytotoxic $\mathrm{T}$ cells in the TME. We further showed that when the PI3K/mTOR dual inhibitor, gedatolisib, was combined with ICI therapy, there was an $\sim 85 \%$ inhibition of growth of PyMT breast tumors. This combinatorial therapy induced durable DC, T-cell and natural killer (NK)-cell responses both systemically and locally in the TME. Notably, the addition of PTX to the gedatolisib + ICI regimen achieved the best response, with complete regression of $60 \%$ of PyMT tumors after 30 days of treatment. 
Analysis of a BC patient dataset from the Single Cell RNA-Seq Expression Atlas suggest that while many PI3K/mTOR-associated genes (e.g., PI3KCA, PI3KCB, MTOR, $R S P 6$, and $4 E-B P 1$ ) are ubiquitously expressed, PI3KCD (encoded for PI3K $\delta$ ) and PIK3CG (encoded for $\mathrm{PI} 3 \mathrm{~K} \gamma$ ) are preferentially expressed in PD-1 $1^{+}$lymphocytes in the primary and metastatic TNBC. Therefore, the combination therapy of gedatolisib + PTX + ICI may translate into successful clinical outcome in BC patients, especially TNBC, and warrants further evaluation.

\section{Results}

2.1. Combination of PI3Ka Isoform-Specific Inhibitor, Alpelisib, and Paclitaxel Induces Granulocyte Responses but Does Not Synergizes with Immunotherapy

The PI3K $\alpha$ inhibitor, alpelisib, has shown some promise for treatment of $\mathrm{ER}^{+}$breast cancer in combination with fulvestrant [13]. In addition, alpelisib combined with CDK4/6 inhibition enhances response to ICI in $\mathrm{pRb}^{+}$triple-negative BCs [14]. Since PI3K $\alpha$ is frequently mutated or activated in $\mathrm{BCs}$, we chose to investigate the effects of alpelisib combined with PTX in response to ICI (anti-PD-1 and anti-CTLA-4) using a luminal, TNBC-like PyMT orthotopic implantation mouse model of breast cancer in C57BL/ 6 mice. Treatment of PYMT tumor-bearing mice with (1) vehicle for alpelisib and vehicle for PTX + IgG, (2) alpelisib (50 mg/kg) + PTX (10 mg/kg) + IgG, (3) vehicles + ICI (200 ug anti-PD-1 and 100 ug anti-CTLA-4), or (4) alpelisib + PTX + ICI was initiated when the tumors were $\sim 125 \mathrm{~mm}^{3}$, and continued for 2 weeks. After 2 weeks of treatment, there was a significant reduction in tumor volume of mice treated with vehicles $+\mathrm{ICI}(p=0.02)$, alpelisib + PTX + $\operatorname{IgG}(p<0.001)$, and alpelisib + PTX + ICI $(p=0.004)$ compared to vehicles + IgG-treated mice (Figure 1a). In addition, a significant reduction in tumor volume was observed in mice treated with alpelisib + PTX + IgG compared to ICI + vehicle-treated mice $(p=0.05)$. However, there was no significant difference in the tumor volume of mice treated with alpelisib + PTX + ICI versus alpelisib + PTX + IgG or with vehicles + ICI, suggesting that the addition of ICI to PI $3 \mathrm{~K} \alpha$ inhibitor plus PTX did not improve the response to ICI alone in mice bearing PyMT tumors.

(a)

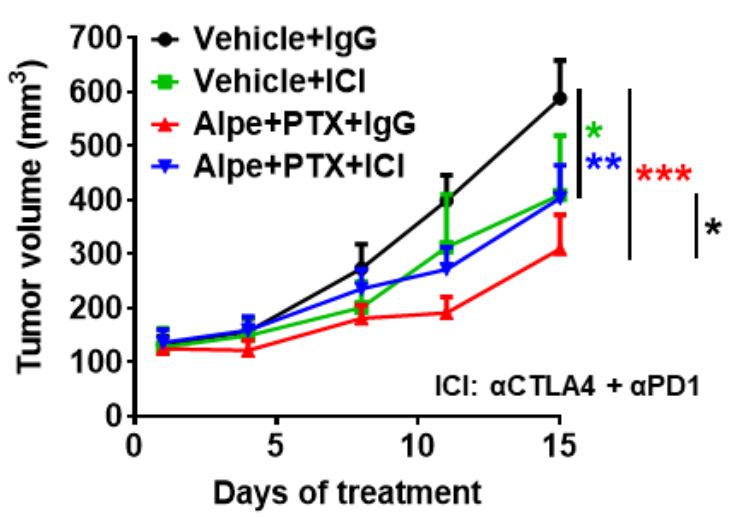

Figure 1. Cont. (b)

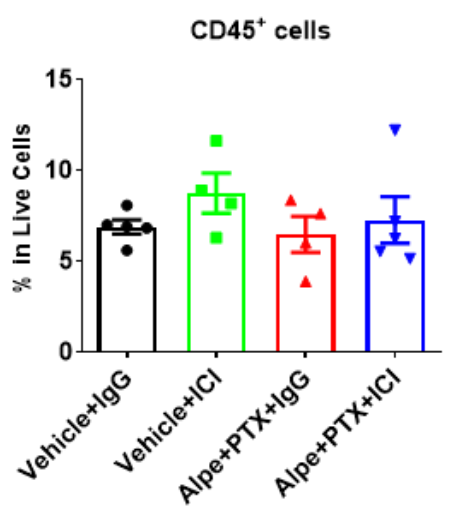


(c)

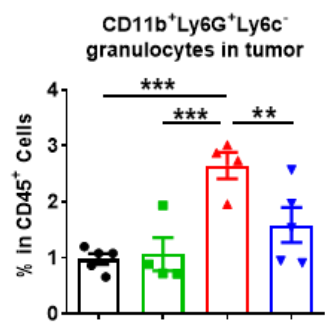

(d)

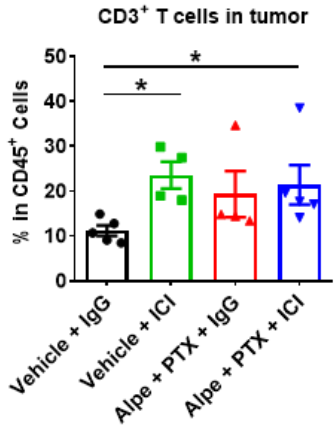

(e) Cell killing percentage of luciferase reporter-bearing breast cancer cells by granulocytes

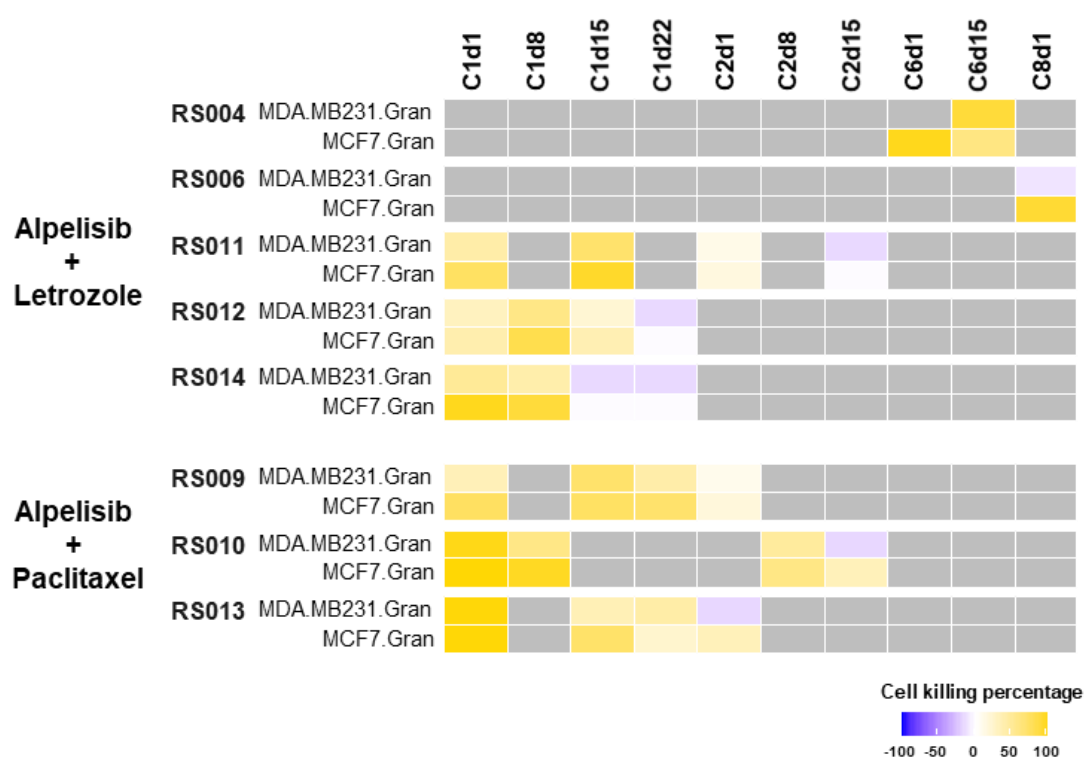

Figure 1. Combination of PI3K $\alpha$ isoform-specific inhibitor, alpelisib, and paclitaxel induces granulocyte responses but did not synergize with immunotherapy. Female C57BL/ 6 mice were injected with 500,000 PyMT cells in mammary fat pads, and treatment with $50 \mathrm{mg} / \mathrm{kg}$ alpelisib (5 days/week, oral), $10 \mathrm{mg} / \mathrm{kg}$ PTX (every 3 days, retroorbital), and ICI (200 ug/mouse anti-PD-1 and $100 \mathrm{ug} /$ mouse anti-CTLA-4 every 3 days, intraperitoneal) was started when tumors reached $125 \mathrm{~mm}^{3}$. (a-d) Primary PyMT tumor samples from mice in each experimental group were collected and immune profiled by flow cytometric analyses ( $\mathrm{n}=5$ per group). (e) Granulocytes (Gran) were isolated and collected by density gradient centrifugation from $250 \mathrm{ul}$ of peripheral blood of patients and co-cultured with breast cancer cells MDA-MB231 and MCF7. A heatmap of percent killing of cancer cells is shown. Data not available are shown in gray cells. $\mathrm{C}$, cycle of treatment. $\mathrm{d}$, days of treatment in the cycle. Where indicated, ${ }^{*} p<0.05 ;{ }^{* *} p<0.01 ;{ }^{* * *} p<0.001$.

To obtain more insight into the immune responses in the tumor microenvironment (TME), multicolor fluorescence-activated cell sorting (FACS) analysis was performed on each tumor to characterize and quantitate the tumor immune infiltrate. There was no significant difference in total CD $45^{+}$leukocyte abundance in the tumors across all treatment groups (Figure 1b), where the gating strategy of immune composition in the TME is shown

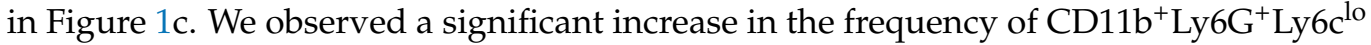
granulocytes in tumors of mice treated with alpelisib + PTX + IgG compared to vehicle + IgG $(p<0.001)$ and vehicle + ICI-treated $(p=0.002)$ mice (Figure $1 \mathrm{~d})$. The addition of ICI to this alpelisib + PTX therapeutic regimen significantly $(p=0.01)$ reduced granulocyte frequency to the levels as in vehicle + IgG-treated tumors. Furthermore, there was a significant increase in T cells of tumors from mice treated with vehicle + ICI $(p=0.04)$ or with alpelisib + PTX + ICI $(p=0.01)$, but not alpelisib + PTX, as compared to vehicle + IgG-treated mice (Figure 1e). However, the addition of alpelisib + PTX to ICI therapy did not further promote a T cell-response in the TME. There were no significant changes in monocytes, macrophages, NK cells, total DC, and T regulatory cells (Tregs) across treatment groups (Supplementary Figure S1). Together, while mice treated with alpelisib + PTX did show inhibition of tumor growth with induced granulocyte responses in the TME, alpelisib + PTX did not increase the sensitivity of PyMT tumors to ICI therapy.

\subsection{Alpelisib Induces Beneficial, but Not Durable, Granulocyte Responses in Breast Cancer Patients}

Evaluation of the leukocyte profile in peripheral blood of patients enrolled in clinical trials (NCT01791478, NCT01872260, or NCT02379247) combining PI3K antagonists alpelisib with letrozole or PTX in our previous work showed that an early cytotoxic T-cell response $\left(\mathrm{CD} 45^{+} \mathrm{CD} 8{ }^{+} \mathrm{CD} 107 \mathrm{a}^{+}\right)$over cycle 1 of treatment significantly correlated with the duration of therapeutic response (months), suggesting that a patient's early cytotoxic 
T-cell response from peripheral blood could serve as a predictive biomarker for therapeutic response to PI3K inhibition [15]. Given that alpelisib + PTX increased the frequency of granulocytes, but not $\mathrm{T}$ cells, in the PyMT tumors, to determine whether our findings regarding alpelisib induction of granulocytes observed in mouse PyMT BC model are recapitulated in human BCs (Figure 1e and Supplementary Figure S2), we further evaluated the granulocyte response on these clinical trials of alpelisib (PI3K $\alpha$ inhibitor) plus letrozole (RS-004, RS-006, RS-011, RS-012 and RS-014) or paclitaxel (RS-009, RS-010 and RS-013), for a period of up to 8 cycles or until withdrawal from the trial. Reasons for going off-trial were depression, progressive disease, or death. While there was no significant alteration of total $\mathrm{CD}_{4} 5^{+}$leukocytes in the peripheral blood after the first cycle of treatment (Supplementary Figure S2a), BC patients who responded to treatment with a positive fold-change in their granulocyte population had an average duration of treatment response of $49.2 \pm 11.9$ months (Supplementary Figure S2b). In contrast, BC patients who responded to treatment with a negative fold-change in their granulocyte population had an average duration of therapeutic response of only $20.25 \pm 14.9$ months. Using multicolor FACS analysis, we showed that there is a positive trend of correlation (correlation coefficient $=0.80, p=0.13$ on cycle 1 day 8 ; correlation coefficient $=0.50, p=0.45$ on cycle 1 day 15) between the early granulocyte $\left(\mathrm{CD} 45^{+} \mathrm{CD} 66 \mathrm{~b}^{+}\right)$population relative to progression free survival (PFS) (months) over cycle 1 of treatment (Supplementary Figure S2c). Furthermore, we isolated granulocytes from patient peripheral blood leukocytes (PBL) and evaluated the functional ability of these granulocytes to kill human ER ${ }^{+} \mathrm{MCF} 7$ and triple-negative MDA-MB231 breast cancer cells ex vivo over the cycles of therapy. Our ex vivo killing assay suggested an effective $60-100 \%$ killing of tumor cells by PBL-sorted granulocytes from peripheral blood of alpelisib plus letrozole or paclitaxel-treated patients over cycle 1 of treatment (Figure 1f). While the PBL-sorted granulocytes of cycle 1-2 were not available for $2 / 5$ patients with alpelisib+letrozole treatment (RS004 and RS006), when we analyzed the PBL-sorted granulocytes from alpelisib + letrozole-treated patients (RS-011, RS-012 and RS-014) and the alpelisib + paclitaxel-treated patients (RS-009, RS-010 and RS-013), we observed these granulocytes gradually lost their anti-tumor effects on tumor cell growth inhibition in the late cycle 1 to cycle 2 of treatments, suggesting that while the alpelisib-induced granulocyte-mediated anti-tumor effects may have potential benefits, the anti-tumor activity of these granulocytes is not maintained.

\subsection{Pan-PI3K Inhibitor, Copanlisib, Plus Immune Therapy Produced Partial Remission of PyMT Breast Tumor Growth}

Given that the combination of PI3K $\alpha$-specific inhibition plus PTX failed to sensitize PyMT tumors for ICI therapy, we postulated that it might be necessary to also inhibit other PI3K isoforms (e.g., PI3K $\gamma$ ) based on the findings that PI3K $\gamma$ or pan-PI3K inhibition can shift the tumor immune microenvironment to one that is anti-tumor by directly affecting macrophage phenotype [10,16]. As noted earlier, severe toxicity issues coupled with poor clinical response resulted in withdrawal of the pan-PI3K inhibitor, BKM120, from the clinic $[10,11]$. However, another pan-PI3K inhibitor, copanlisib, has been FDA approved for treatment of relapsed follicular lymphoma, and its toxicity effects are tolerable [17]. Therefore, we sought to determine whether combined treatment with copanlisib + PTX plus ICI would be effective for treatment of PyMT breast cancer in the preclinical model. As shown in Figure 2a, after 3 weeks of treatment, there were significant decreases in tumor volume of mice treated with copanlisib + PTX $+\operatorname{IgG}(p=0.001)$, vehicle + ICI $(p=0.001)$, and copanlisib + PTX + ICI $(p<0.001)$ compared to vehicle + IgG-treated mice. In addition, there was a significant decrease in tumor volume of mice treated with copanlisib + PTX + ICI versus copanlisib + PTX + IgG-treated $(p=0.02)$ mice, suggesting that the addition of ICI enhanced response to copanlisib + PTX; however, the addition of copalisib + PTX did not enhance the response to ICI alone. 
(a)

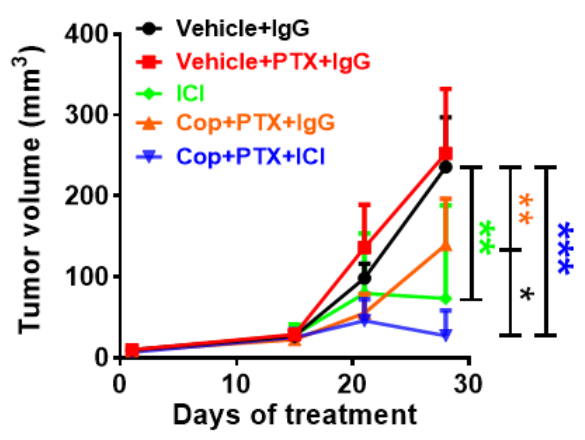

(c)

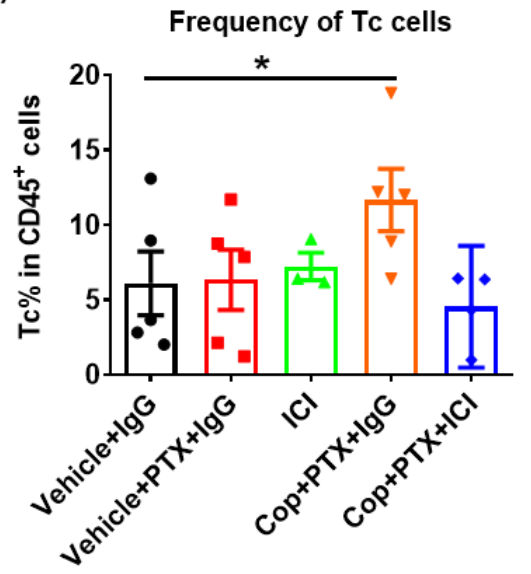

(b)

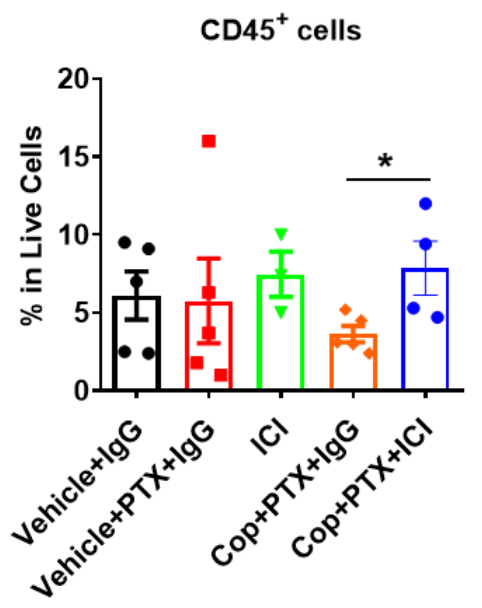

(d)

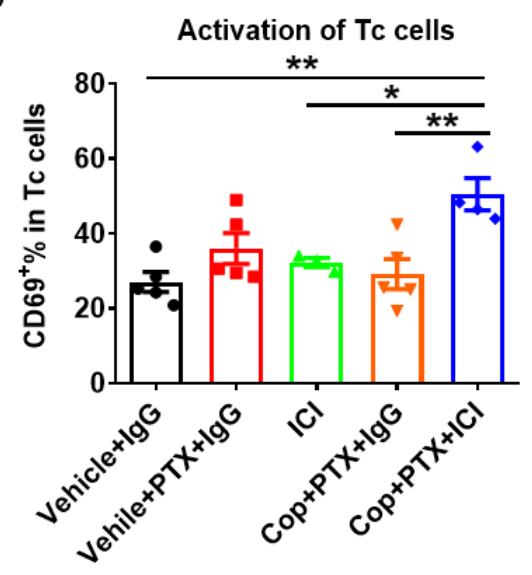

(e)

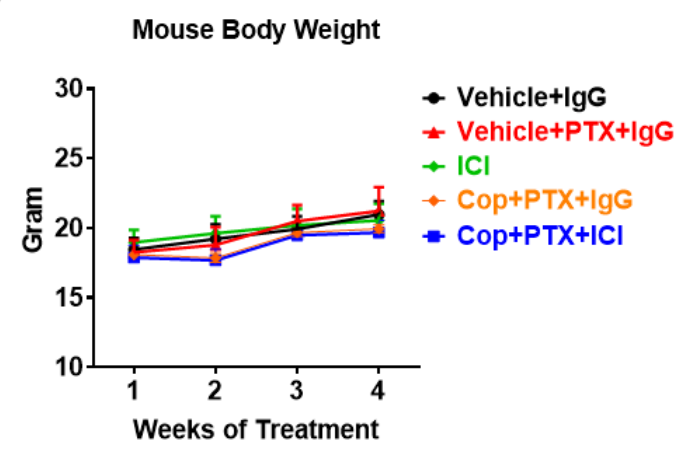

Figure 2. Pan-PI3K inhibitor, copanlisib, plus immune therapy produced partial remission of PyMT breast tumor growth. Female C57BL/ 6 mice were injected with 100,000 PyMT cells in the $4^{\text {th }}$ mammary fat pads. (a) Tumor measurements were recorded throughout the course of treatment to produce tumor growth curve, and treatments with $0.8 \mathrm{mg} / \mathrm{kg}$ copanlisib (every 2 days, intravenous), $10 \mathrm{mg} / \mathrm{kg}$ PTX (every 3 days, retroorbital), and ICI (200 ug anti-PD-1 and 100 ug anti-CTLA-4 every 3 days, intraperitoneal) were started 1 week post tumor cell implantation. Tumor samples were collected from each treatment group at day 28 of treatment and immune profiled by FACS analysis to measure (b) all immune cells and (c,d) immune cell subpopulations in the tumor. (e) Mouse body weight recorded for 28 days of treatment. (All graphs show mean \pm SEM $\mathrm{n} \geq 4$ per group.) Where indicated, ${ }^{*} p<0.05 ;{ }^{* *} p<0.01$. 
The immune infiltrate of each tumor was determined by multicolor FACS analysis. While there were no significant differences in total $\mathrm{CD} 45^{+}$tumor-infiltrated leukocyte (TIL) abundance in tumors across all treatment groups compared to vehicle $+\operatorname{IgG}$, the addition of ICI into copanlisib + PTX increased the frequency of TIL in the TME $(p=0.04)$ (Figure 2b). Further characterization of immune cell subpopulations indicated a significant increase in the $\mathrm{CD} 8^{+}$cytotoxic $\mathrm{T}$ cell $(\mathrm{Tc})$ content in tumors of mice treated with copanlisib + PTX $+\operatorname{IgG}(p=0.02)$ as compared to tumors from mice treated with vehicle + $\operatorname{IgG}$ (Figure 2c). In addition, copanlisib + PTX + ICI increased the abundance of activated $\left(\mathrm{CD} 69^{+}\right)$Tc cells as compared to tumors from mice treated with vehicle $+\operatorname{IgG}(p=0.002)$, vehicle + ICI $(p=0.048)$, or copanlisib + PTX $+\operatorname{IgG}(p=0.006)$ (Figure $2 \mathrm{~d})$. There were no significant changes in granulocyte, monocyte, macrophage, or natural killer cell content of tumors in response to treatments. These data suggest that pan-PI3K inhibition by copanlisib, combined with PTX, enhanced Tc cell responses and promoted the activation of Tc cells that enhanced the response of BC to ICI therapy. Therefore, as we demonstrated previously with BKM-120 [10], pan-inhibition of the PI3K pathway may indeed be effective in enhancing response to ICI therapy. However, the effectiveness of this combination, while tolerable and without loss of mouse body weight (Figure 2e), is limited to a partial remission. mTOR is another signal transducer that acts downstream of PI3K activation. We postulated that an inhibitor that inhibited both mTOR and PI3Ks might be more effective that PI3K inhibition alone when combined with ICI therapy for breast cancer treatment.

\subsection{Dual PI3K/mTOR Inhibitor, Gedatolisib, Plus Immune Therapy Effectively Stopped PyMT Breast Tumor Growth}

Gedatolisib is a potent and reversible inhibitor of PI3Ks and mTOR. IC50 values for PI3K $\alpha, \mathrm{PI} 3 \mathrm{~K} \beta, \mathrm{PI} 3 \mathrm{~K} \delta, \mathrm{PI} 3 \mathrm{~K} \gamma$, and $\mathrm{mTOR}$ are $0.4 \mathrm{nmol} / \mathrm{L}, 6 \mathrm{nmol} / \mathrm{L}, 8 \mathrm{nmol} / \mathrm{L}, 6 \mathrm{nmol} / \mathrm{L}$, and $10 \mathrm{nmol} / \mathrm{L}$, respectively [18]. To develop mechanistic insight into how gedatolisib might enhance sensitivity to ICI therapy in preclinical models, PyMT cells $\left(1 \times 10^{5}\right)$ were injected into the fourth mammary fat pad equivalent on either flank of 6-8-week-old female C57BL/ 6 mice. Treatments were as follows: (1) vehicle for gedatolisib + IgG (300 $\mu \mathrm{g})$, (2) gedatolisib $(12 \mathrm{mg} / \mathrm{kg})+\mathrm{IgG}(300 \mu \mathrm{g})$, (3) vehicle for gedatolisib + ICI (200 ug anti-PD-1 and $100 \mathrm{ug}$ anti-CTLA-4), or (4) gedatolisib (12 mg/kg) + ICI (200 ug anti-PD-1 and $100 \mathrm{ug}$ anti-CTLA-4). Treatments were initiated when the tumors were $\sim 150 \mathrm{~mm}^{3}$ and continued for 17 days. We sampled the tumors at early (day 10 post-treatment) and late (day 17 posttreatment) stages. There was a significant reduction in tumor volume in mice treated with gedatolisib + ICI $(p=0.005)$ compared to vehicle + IgG-treated mice, while no significant change of tumor volume was observed in monotherapy treatments (Figure 3a). Importantly, there was a significant reduction in tumor volume of mice treated with gedatolisib + ICI compared to tumors from mice treated with vehicle + gedatolisib $(p=0.025)$ or vehicle + ICI $(p=0.020)$. Consistent with the tumor volume results, gedatolisib + ICI reduced the tumor weight at day 10 and 17 compared to the tumors from mice treated with vehicle $+\operatorname{IgG}$ or monotherapy (Figure $3 b$ ). Notably, gedatolisib + ICI completely stopped tumor growth for the entire treatment period with $\sim 85 \%$ growth inhibition compared to the vehicle + IgG-treated tumors. 
(a)

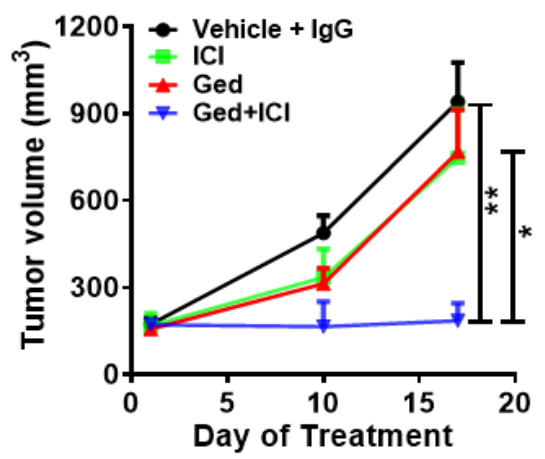

(c)

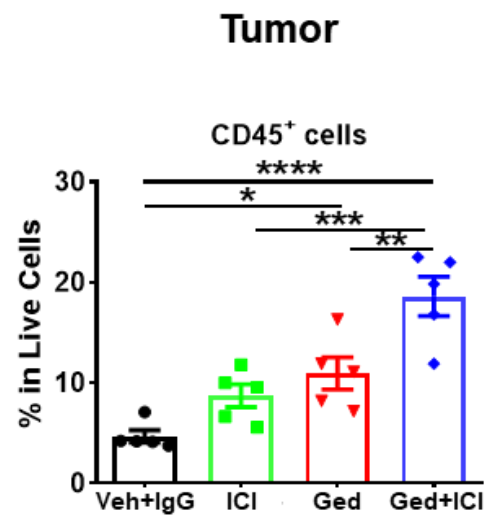

(b)

Day 10

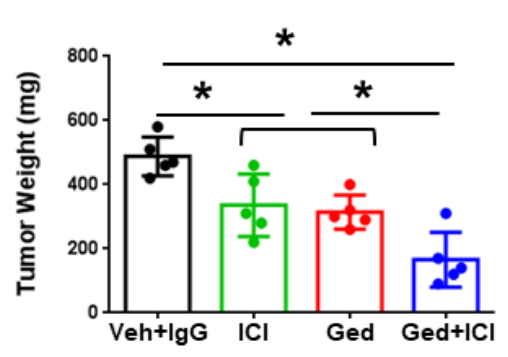

Day 17

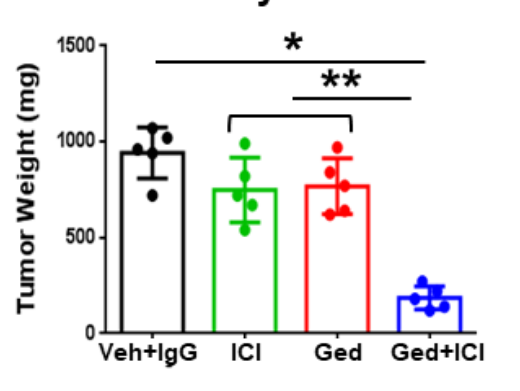

Bone Marrow

$\mathrm{CD} 5^{+}$cells

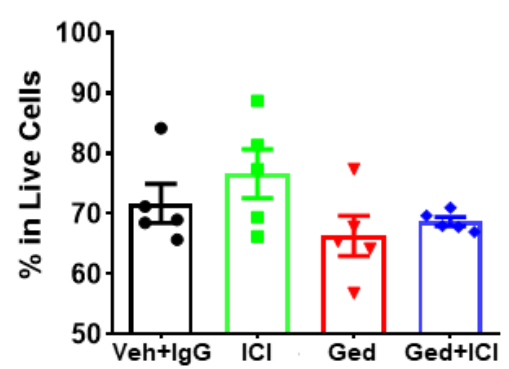

\section{Blood}

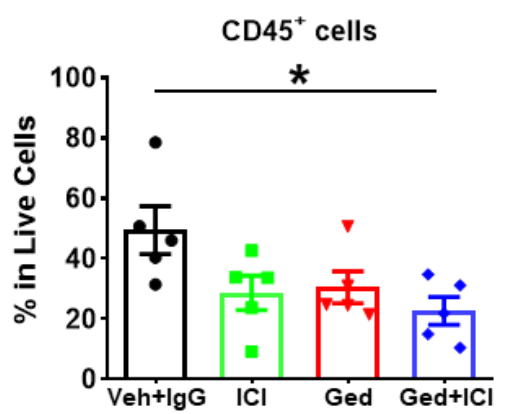

Figure 3. PI3K/mTOR inhibitor, gedatolisib, plus immune therapy completely restrained PyMT breast tumor growth. (a-c) Female C57BL/ 6 mice were injected with 500,000 PyMT cells in the 4th mammary fat pad. Mice were treated with immune therapy (CTLA4 at $100 \mathrm{ug} /$ mouse and PD-1 Abs at $200 \mathrm{ug} /$ mouse every 3 days, intraperitoneal) and/or gedatolisib (12 mg/kg, twice a week, intravenous), and vehicle plus isotype IgG antibodies were treated as control. Treatments were started when tumors reached $150 \mathrm{~mm}^{3}$. Tumor weights were measured at days 10 and 17. Primary tumor, bone marrow (BM) and lung samples were collected at day 10 post treatment and immune profiled by flow cytometric analyses ( $\mathrm{n}=5$ per group). Where indicated, ${ }^{*} p<0.05 ;{ }^{* *} p<0.01$; ${ }^{* * *} p<0.001$; and ${ }^{* * *} p<0.0001$.

Immune profiling of local (TME) and systemic (bone marrow [BM] and peripheral blood) responses were conducted by multi-color FACS analysis (Figure 3c). Treatment of gedatolisib + IgG $(p=0.03)$ resulted in a significant increase in the CD45 $5^{+}$TIL average frequency from $4.7 \%$ to $10.94 \%$ in all live cells in the TME compared to vehicle + IgG treatment. Importantly, the combination of gedatolisib + ICI further increased the abundance of CD $45^{+}$ TIL to $18.61 \%$ compared to vehicle + ICI $(8.7 \%)(p=0.0008)$, gedatolisib + IgG $(10.94 \%)$ $(p=0.007)$ or vehicle $+\operatorname{IgG}(4.7 \%)(p<0.0001)$ treatments. There were no significant changes in the abundance of CD $45^{+}$leukocytes in the BM, while a $\sim 30 \%$ reduction was observed in the peripheral blood in the mice treated with gedatolisib + ICI $(p=0.03)$ compared to vehicle $+\operatorname{IgG}$. These results suggest that the gedatolisib + ICI-induced immune cell infiltration into tumors likely resulted from the increased recruitment of leukocytes from blood circulation into the TME, and did not result from de novo hematopoiesis in the BM. 
FACS analyses were performed to characterize immune cell subpopulations in tumors at early (10 days) and late (17 days) treatment stages (Figure $4 a)$. At the early treatment timepoint, there was a significant induction of $\mathrm{CD} 4^{+} \mathrm{T}$ cell content in the tumors with treatments of vehicle + ICI $(13.7 \%, p=0.01)$, gedatolisib + IgG $(19.6 \%, p=0.0002)$, or gedatolisib + ICI $(25.5 \%, p<0.0001)$, compared to vehicle $+\operatorname{IgG}(8.6 \%)$. While vehicle + ICI $(6.9 \%, p=0.743)$ did not maintain the increased level of $\mathrm{CD} 4{ }^{+} \mathrm{T}$ cells at the late treatment timepoint, gedatolisib $+\operatorname{IgG}(19.2 \%, p=0.014)$ or gedatolisib $+\mathrm{ICI}(12.4 \%, p=0.041)$ resulted in a durable $\mathrm{CD}^{+} \mathrm{T}$-cell response as observed in the late treatment timepoint, compared to vehicle $+\operatorname{IgG}(7.4 \%)$. In addition, there was a significant induction of $\mathrm{CD} 8^{+} \mathrm{T}$ cells at the late treatment timepoint in the tumors treated with gedatolisib $+\operatorname{IgG}(24.5 \%, p=0.02)$ or gedatolisib + ICI $(22.8 \%, p=0.05)$ compared to vehicle + IgG $(13.6 \%)$. Accompanied with the treatment-induced T-cell responses, the frequency of DCs in the TME was promoted at the early timepoint of treatments with vehicle + ICI $(13.14 \%, p=0.02)$, gedatolisib + $\operatorname{IgG}(15.36 \%, p=0.0002)$, or gedatolisib + ICI $(14.88 \%, p<0.0001)$, compared to vehicle $+\operatorname{IgG}(9.0 \%)$. Besides increased frequency of T cells and DCs in the TME, gedatolisib + ICI treatment also induced a 1.5-2-fold increase in the activation $\left(\mathrm{CD} 69^{+}\right)$of $\mathrm{CD} 8^{+}$Tc cells (Figure $4 \mathrm{~b}, p=0.0219$ at day 10, $p=0.0198$ at day 17) and NK cells (Figure $4 \mathrm{c}, p=0.0050$ at day $10, p=0.0308$ at day 17) compared to vehicle $+\operatorname{IgG}$ at both early and late treatment timepoints. There was a clear induction of granulocytic population and M2 macrophages in the tumors at day 17 compared to day 10, which was independent of therapy types and could be a potential mechanism of resistance in the residual tumors.

(a)

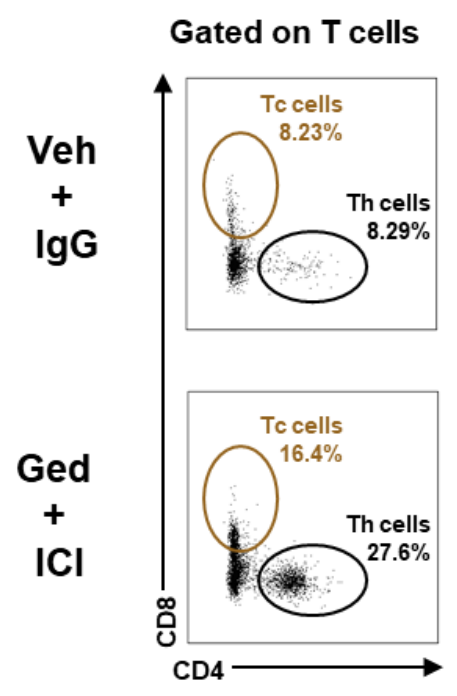

\section{Frequency of Immune cell subsets}

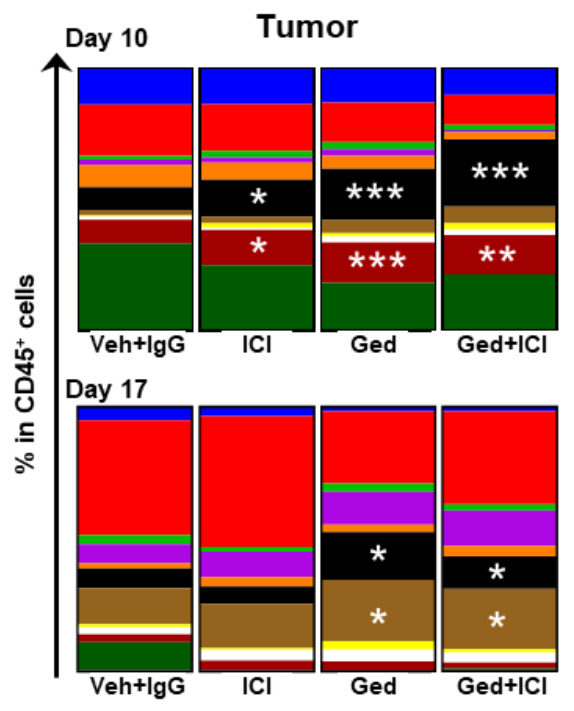

Figure 4. Cont. 
(b)

\section{Activation of $\mathrm{CD}^{+}$Tc cells}
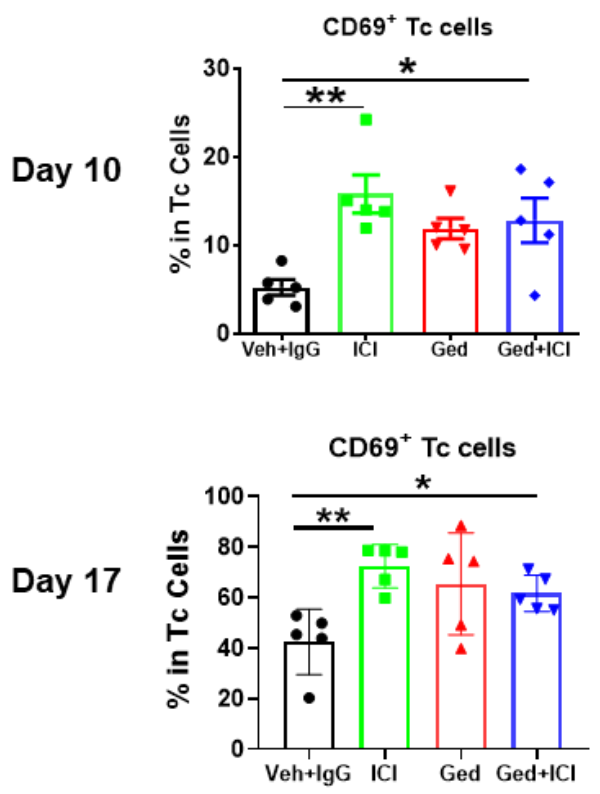

(c)

\section{Activation of NK cells}
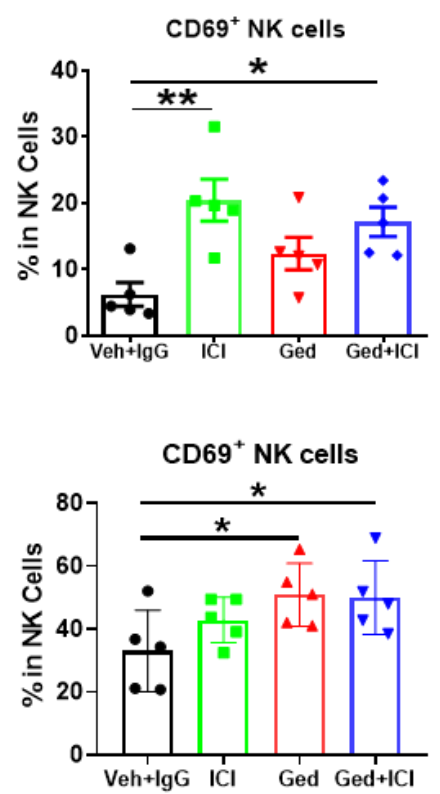

Figure 4. Gedatolisib + Immune induces durable local DC, T-cell and NK-cell responses. (a-c) Female C57BL/6 mice were injected with 500,000 PyMT cells in fat pad. Mice were treated with immune therapy (CTLA4 at $100 \mathrm{ug} / \mathrm{mouse}$ and PD-1 Abs at $200 \mathrm{ug} / \mathrm{mouse}$ every 3 days, intraperitoneal) and/or gedatolisib (12 mg/ kg, twice a week, intravenous), and vehicle plus isotype IgG antibodies were treated as control. Treatments were started when tumors reached $150 \mathrm{~mm}^{3}$. Immune profiling of primary tumor samples was analyzed by flow cytometry ( $\mathrm{n}=5$ per group). Where indicated, ${ }^{*} p<0.05 ;{ }^{* *} p<0.01$.

Next, we conducted multicolor FACS analysis on systemic immune subpopulations in the BM and peripheral blood (Supplementary Figure S3a). Consistent with the TME immune profiling results, there was a significant increase in $\mathrm{CD} 8^{+} \mathrm{Tc}$ cells in the $\mathrm{BM}$ of mice treated with gedatolisib + ICI (early timepoint, from $5.2 \%$ to $11.6 \%$; late timepoint, from $5.7 \%$ to $17.0 \%$ ), but not gedatolisib monotherapy, compared to vehicle + IgG. Interestingly, the gedatolisib + ICI-induced CD8 ${ }^{+}$Tc-cell response is associated with a reduction in granulocytes in the BM. In the blood circulation, there were significant reductions in granulocytes at early treatment timepoint and an induction of monocytes at the late treatment timepoint across all treatment groups compared to vehicle + IgG. Notably, gedatolisib + IgG $(15 \%)$ and gedatolisib + ICI $(20 \%)$ resulted in a 1.5-fold and 2-fold increase in circulating $\mathrm{MHCII}^{+} \mathrm{CD} 11 \mathrm{c}^{+} \mathrm{DC}$ in the peripheral blood compared to the vehicle $+\mathrm{IgG}$ $(5 \%)$ at the early (Supplementary Figure S3b), but not late (Supplementary Figure S3c), treatment timepoints. Taken together, these results suggested that the combination of gedatolisib and ICI induces durable local and systemic DC and T-cell immune responses in PyMT breast cancer tumors.

\subsection{Gedatolisib Plus PTX and Immune Therapy Resulted in $60 \%$ Complete Regression of PyMT Breast Tumors}

While the combination treatment of gedatolisib and ICI suppressed PyMT tumor growth, no tumors completely regressed. Recently, gedatolisib was reported to have a tolerable safety profile when combined with PTX in a phase I dose-escalation study with advanced ovarian, endometrial, and non-small cell lung cancers [19]. We further investigated whether the addition of PTX to the gedatolisib treatment might enhance sensitivity to ICI therapy in the PyMT model. After 4 weeks of treatment, there was a significant reduction in tumor volume (Figure $5 a$ ) and tumor weight (Figure $5 b$ ) in mice treated with vehicle + ICI, gedatolisib + PTX + IgG and gedatolisib + PTX + ICI, compared 
to vehicle + IgG-treated mice. Impressively, $4 / 6$ tumors from mice treated with gedatolisib + PTX + ICI completely regressed, while the remaining $2 / 6$ tumors exhibited an $85 \%$ growth inhibition compared to the vehicle + IgG-treated tumors. Furthermore, gedatolisib + PTX + ICI introduced mild toxicity in the first week of treatment based on an $10 \%$ loss of mouse body weight, which recovered afterwards (Figure 5c), consistent with the tolerable safety profile of gedatolisib + PTX in patients [19].

The regression of tumors in the gedatolisib + PTX + ICI treatment group made it impossible to ascertain the effect of treatment on specific subpopulations of immune cells in the TME. To better understand the anti-tumor immune response to this drug regimen, we immune-profiled the peripheral blood samples after 28 days of treatment in each mouse (Figure 5d). Gedatolisib + PTX + ICI, but not gedatolisib + PTX + IgG or vehicle $+\mathrm{ICI}$, significantly promoted $\mathrm{MHCII}{ }^{+} \mathrm{CD} 11 \mathrm{c}^{+} \mathrm{DC}$ abundance compared to vehicle $+\mathrm{IgG}$ $(p<0.0001)$, gedatolisib + PTX $+\operatorname{IgG}(p=0.0008)$, or vehicle + ICI $(p=0.0007)$. Furthermore, vehicle $+\operatorname{ICI}(p=0.0006)$ or gedatolisib + PTX $+\operatorname{IgG}(p=0.0002)$ significantly increased the frequency of $\mathrm{CD} 9^{+} \mathrm{CD} 8^{+}$activated Tc cells to $\sim 31 \%$ compared to $11 \%$ in the vehicle + IgG-treated mice. Notably, the addition of gedatolisib + PTX into ICI therapy (49\%, $p=0.0091)$ resulted in a further induction of $\mathrm{CD} 9^{+} \mathrm{CD} 8^{+}$activated Tc cells in the blood circulation compared to vehicle + ICI. These data show that dual inhibition of PI3K/mTOR plus PTX shifts the immune cell content of tumors to reverse the "cold" immune phenotype and increases sensitivity to ICI therapy.

(a)

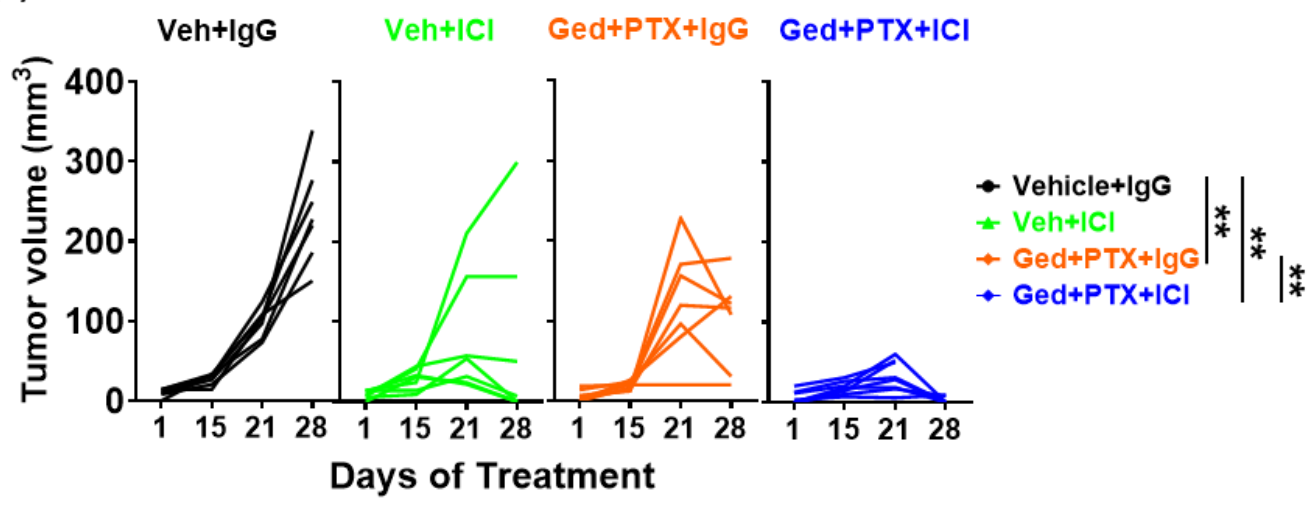

(b)

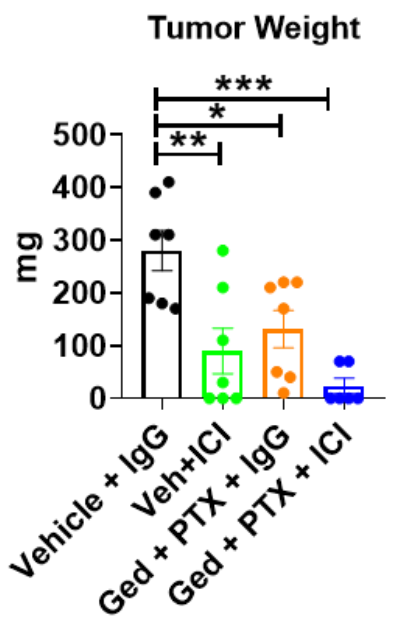

(c)

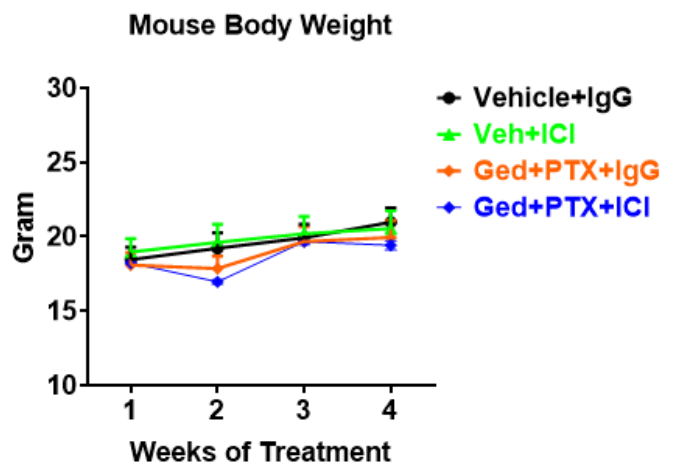

Figure 5. Cont. 
(d)

\section{Peripheral blood immune profile}

CD11C ${ }^{+} \mathrm{MHCII}{ }^{+} \mathrm{DCs}$

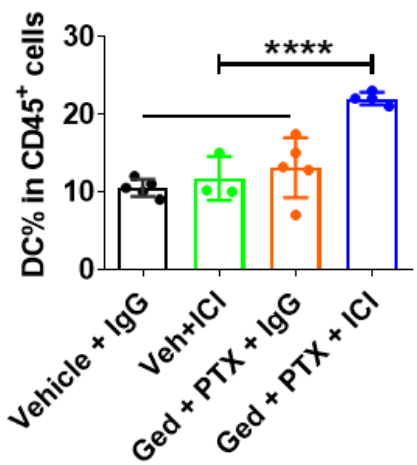

CD69+ Tc cells

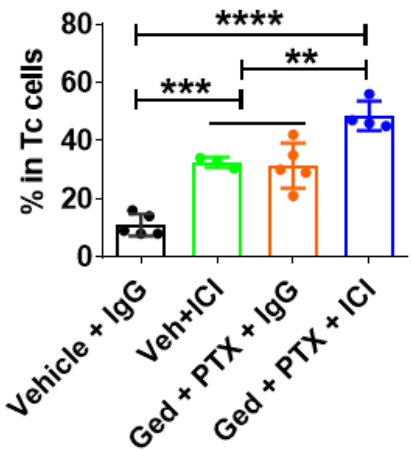

Figure 5. Gedatolisib + PTX + immunotherapy resulted in complete regression of $60 \%$ of the PyMT breast tumors. Female C57BL/6 mice were injected with 500,000 PyMT cells in the 4th mammary fat pad. Mice were treated with immune therapy (CTLA4 at $100 \mathrm{ug} /$ mouse and PD-1 Abs at $200 \mathrm{ug} /$ mouse every 3 days, intraperitoneal) and/or gedatolisib (12 mg/ $\mathrm{kg}$, twice a week, intravenous), $10 \mathrm{mg} / \mathrm{kg}$ PTX (every 3 days, retroorbital), and vehicle plus isotype IgG antibodies were treated as control. Treatments were started 1 week post tumor cell implantation and mouse body weight was monitored. Tumor weights were measured at day 28 . Peripheral blood samples at 28 days post-treatment were analyzed by flow cytometry. $(\mathbf{a}-\mathbf{c}), \mathrm{n} \geq 7$ per group; $(\mathbf{d})$, $\mathrm{n}=3-5$ per group. Where indicated, ${ }^{*} p<0.05 ;{ }^{* *} p<0.01 ;{ }^{* * *} p<0.001$; and ${ }^{* * * *} p<0.0001$.

2.6. PIK3CD and PIK3CG Are Preferentially Expressed in the Lymphocytes of Triple-Negative BC Patient Tumors

Molecular analysis with gene expression profiling showed that $\mathrm{BC}$ could be subclassified into different subtypes: luminal ER-positive (luminal A and luminal B), HER2enriched, and triple-negative or basal-like BCs $[20,21]$. To explore the relative gene expression of PI3K/mTOR-associated genes in BC subtypes, we analyzed the publicly available single-cell (sc) RNA-seq dataset from Single Cell Expression Atlas (https:/ /www.ebi.ac. $\mathrm{uk} / \mathrm{gxa} / \mathrm{sc} /$ home) (accessed on 7 December 2020) containing 515 cells from 11 BC patients representing the four subtypes (Figure 6). While PI3KCA (encoded for PI3K $\alpha$ ) and PI3KCB (encoded for PI3K $\beta$ ) are generally expressed among all four BC subtypes, PI3KCD (encoded for PI3K $\delta$ ) and PIK3CG (encoded for PI3K $\gamma$ ) are preferentially expressed in the primary and metastatic TNBC. The mTOR pathway-related genes, including MTOR, RSP6, and 4E-BP1, are ubiquitously expressed in $B C$ subtypes. Further t-SNE analyses suggested that the cells that expressed high levels of PI3KCD and PIK3CG are CD45 ${ }^{+} \mathrm{CD} 3^{+} \mathrm{T}-$ or $\mathrm{CD} 45^{+} \mathrm{CD} 19^{+}$ B- lymphocytes, but not $\mathrm{CD}_{11 \mathrm{~b}^{+}}$myeloid cells. PD-1 is expressed on activated CD4 ${ }^{+}$ and $\mathrm{CD}^{+} \mathrm{T}$ cells and the interaction of PD-1 with its ligands (PD-L1/L2) results in the suppression of T-cell activity [22]. Interestingly, those PI3KCD ${ }^{\text {hi }} P I K 3 C G^{\text {hi }}$ lymphocytes also exhibited a high level of $P D-1$ mRNA expression, suggesting an early T-cell exhaustion phenotype and highlighting a potential beneficial effect of PI3K $\gamma / \delta$ inhibition in TNBC. 


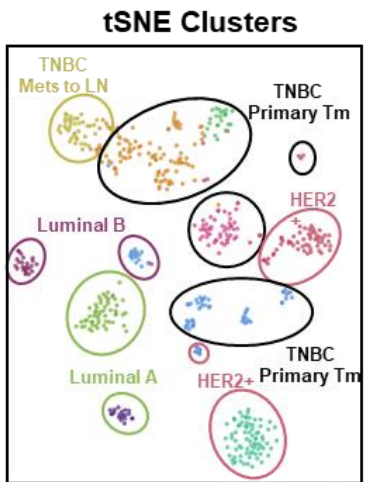

PIK3CA

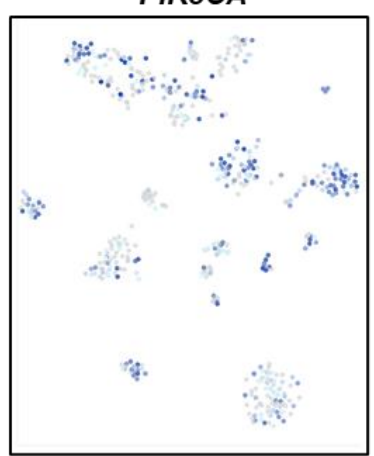

MTOR

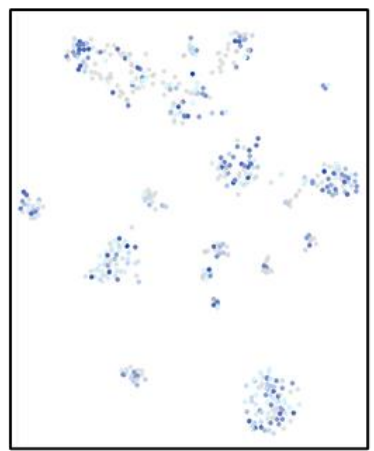

CD11b (ITGAM)

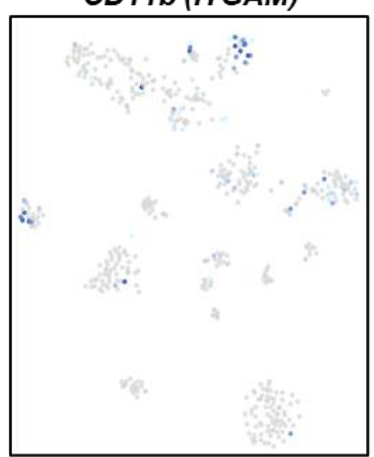

ER (ERS1)

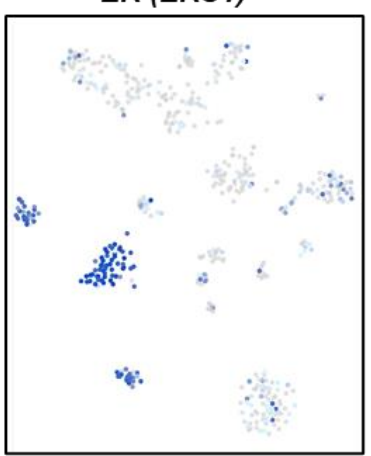

PIK3CB

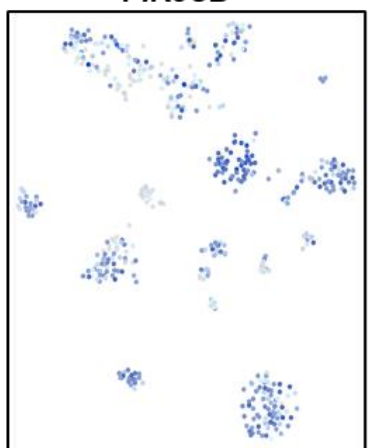

RPS6

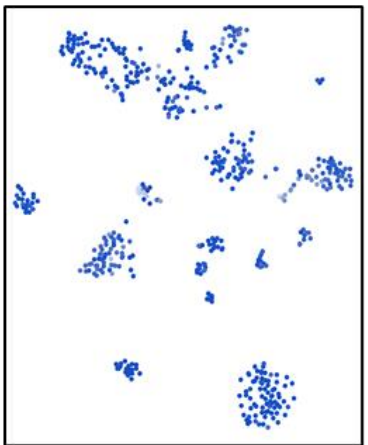

CD3D

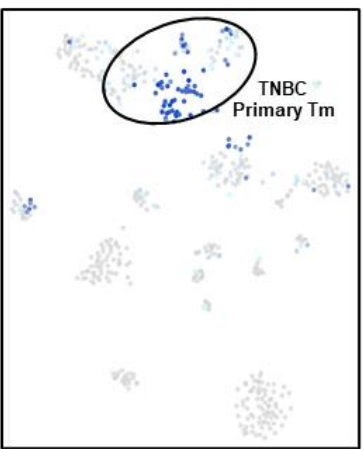

PR (NR3C3)

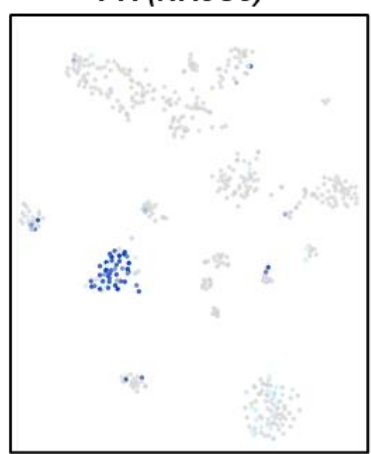

PIK3CD

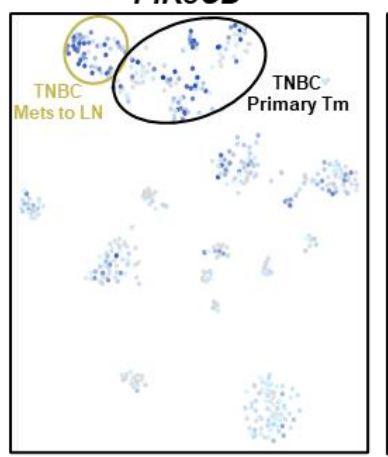

4E-BP1

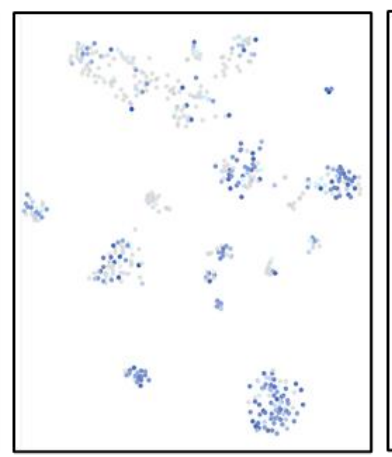

CD19

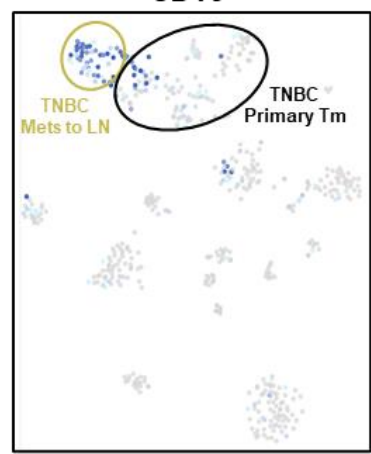

Expression level (CPM)

$\begin{array}{lllll}0 & 10 & 100 & 1 \mathrm{k} & 10 \mathrm{k}\end{array}$

HER2 (ERBB2)

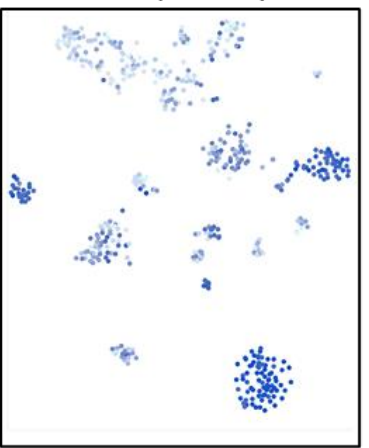

PIK3CG

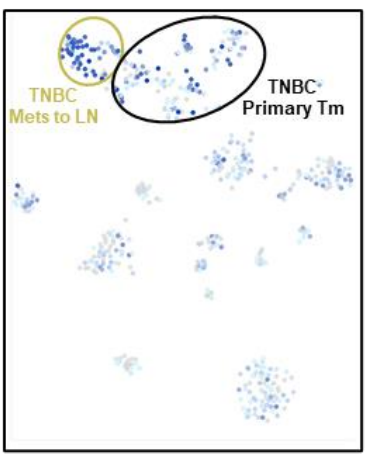

CD45 (PTPRC)

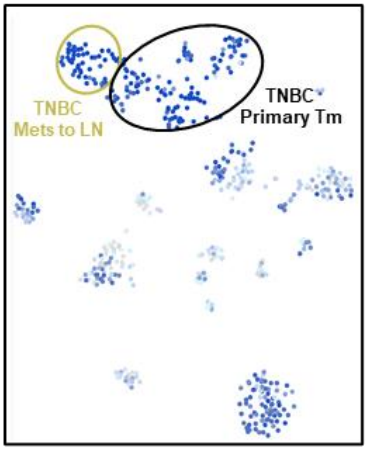

PD-1 (CD279)

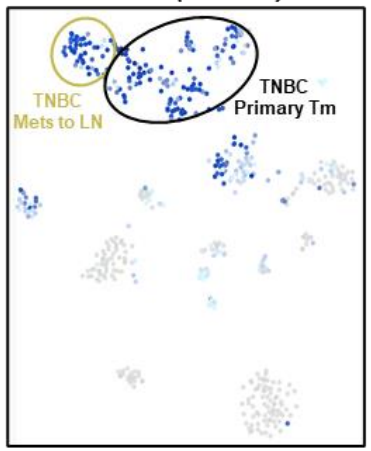

Figure 6. PIK3CD and PIK3CG are preferentially expressed in the lymphocytes of TNBCs. Results of tSNE and gene expression profile were retrieved from Single Cell Expression Atlas (https://www.ebi.ac.uk/gxa/sc/experiments/EGEOD-75688/) (accessed on 7 December 2020). Analysis of scRNAseq from breast cancer cells from primary and lymph node metastases from 11 patients representing the four subtypes of breast cancer: luminal A, luminal B, HER2, and triple-negative breast cancer. Number of cells: 539. t-SNE perplexity $=25$, color plot by: $\mathrm{K}=10$. 


\section{Discussion}

In this study, we show that it is essential to simultaneously inhibit PI3K isoforms to effectively enhance response to PTX and ICI therapy in the PyMT tumor model. PI3K $\alpha$ inhibition by alpelisib plus PTX did reduce PyMT tumor volume but did not sensitize the tumors to ICI. Evidenced in both our preclinical animal model and clinical trials, alpelisib + PTX induced beneficial, but not durable, granulocyte anti-tumor responses. As a major cellular component of granulocytes, neutrophils can be polarized and display different phenotypes spreading from an antitumor (N1-like) to a pro-tumor (N2-like) phenotype [23,24]. Recent studies in experimental models have provided extensive support for the existence of pro-angiogenic (N2) or anti-tumor (N1) neutrophil phenotypes [23]. The N1 neutrophils are able to generate reactive oxygen species (ROS) and release various pro-inflammatory proteins from intracellular granules, which include myeloperoxidase, lactoferrin, defensin, lysozyme, and proteases (e.g., elastase and gelatinase). These factors can cause tissue damage and tumor cell lysis, as well as potential DNA damage [25]. However, while the neutrophil-driven innate immune response is fast and generally effective, it lacks specificity and the capacity to build long-lived immunological memory compared to the adaptive immune responses, such as T-cell responses. The preferential induction of $\mathrm{T}$ cells by the treatment of PI3K/mTOR inhibitor gedatolisib plus PTX, compared to transiently induced granulocytes by PI3K $\alpha$ inhibitor plus PTX, may partially explain why gedatolisib + PTX better sensitized the tumors for ICI therapy.

Since PI $3 K \alpha$ is often constitutively activated in breast tumor cells, its inhibition should have inhibited PyMT tumor growth but have limited effects on the immune cells, while PI3K $\gamma$ and PI3K $\delta$ are the major PI3Ks expressed in leukocytes. Studies have shown that the inhibition of PI3K $\delta$ reduces myeloid-derived suppressor cells (MDSC) and Tregs [26], and the inhibition of PI3K $\gamma$ reduces MDSC and shifts the macrophages in the TME toward an anti-tumor M1-like phenotype [16,27]. Thus, it is not surprising that others have shown that $\mathrm{PI} 3 \mathrm{~K} \alpha, \delta$ inhibition significantly reduces tumor growth in $4 \mathrm{~T} 1, \mathrm{MC}-38$, and CT-26 tumors by reducing tumor-infiltrating Tregs, MDSCs, and protumor macrophages, while increasing $\mathrm{CD}^{+}$Tc-cell activation and memory [28]. Treatment with the FDA-approved pan-PI3K inhibitor copanlisib plus PTX also resulted in a significant reduction in PyMT tumor growth. ICI removes inhibitory signals of T-cell activation, which enables tumorreactive $\mathrm{T}$ cells to overcome regulatory mechanisms and mount an effective antitumor response $[4,29,30]$. When combined with ICI therapy, there was a significant increase in the immune cell infiltration into tumors, with increased activation of $\mathrm{CD} 8^{+} \mathrm{Tc}$ cells.

Our previous and current data suggest that while pan-PI3K inhibition enhances response to ICI therapy in mammary tumors, the limited efficacy does not override the significant toxicity of these inhibitors in the clinic. In contrast, gedatolisib is reasonably welltolerated and functions essentially as a PI3K/mTOR inhibitor [19]. The mTOR inhibitor everolimus has been previously used to treat breast cancers, but toxicity is a major issue with this drug and many patients stop treatment due to this issue [12]. Here, we utilized a $\mathrm{PI} \mathrm{K} / \mathrm{mTOR}$ dual inhibitor with reduced toxicity as an alternative way to inhibit multiple forms of PI3K and enhance response to ICI therapy. Our data suggest that treatment with gedatolisib + PTX increases sensitivity to ICI therapy. However, our study has limitations. Firstly, in our animal studies, we utilized only one breast cancer model, the PyMT model, which exhibits constitutive activation of the PI3K pathway [31]. Nevertheless, our findings in the mouse model are supplemented with data analysis from human studies, though the number of patients analyzed was small. Clearly additional trials will be needed. Secondly, we utilized $\alpha$ CTLA $4+\alpha$ PD1 as the ICI therapy in preclinical studies; future work is needed to evaluate the feasibility of combining gedatolisib + PTX with $\alpha$ PD1 or $\alpha$ CTLA4 alone to potentially reduce the ICI-induced immune-related adverse events. Lastly, IMpassion130 reported that $\alpha$ PD-L1 antibody atezolizumab in combination of nab-paclitaxel significantly improved PFS and overall survival (OS) of patients with unresectable locally advanced or metastatic TNBC [8]. However, the primary results from IMpassion131 suggested that atezolizumab in combination with PTX did not improve PFS or OS versus placebo + PTX 
in TNBC while the cellular or molecular mechanisms are not clear [32]. In the present study, we showed that PTX monotherapy did not inhibit tumor growth in PyMT preclinical mouse models (Figure 2a). The combination of PTX with PI3K/mTOR inhibitor gedatolisib exhibited a delayed response after 21 days of treatment and the further addition of $\alpha$ CTLA4 $+\alpha \mathrm{PD} 1$ advanced the therapeutic response from onsite (Figure 5a). It is intriguing to explore whether the backbone of PTX (nab-paclitaxel versus PTX) and/or the classes of ICI ( $\alpha$ PDL1 versus $\alpha$ PD1 $+/-\alpha$ CTLA4) would impact the therapeutic outcomes. Given that new combinations of the inhibitors of the $\mathrm{PI} 3 \mathrm{~K} / \mathrm{AKT} / \mathrm{mTOR}$ signaling pathway could provide a new perspective for the management of breast cancers, especially for the cases with precision medicine and drug resistance, future work is need to explore the therapeutic potential and safety profile of mTORC1 inhibitors (e.g., everolimus, temsirolimus and ridaforolimus), mTORC1/2 inhibitors (e.g., vistusertib), as well as AKT inhibitors (e.g., ipatasertib) monotherapy and in combination with ICI.

Current clinical trials of BC are combining gedatolisib with a range of other therapies, including herzuma (NCT03698383), PTK7-ADC (NCT03243331), palbociclib (NCT02626507 and NCT02684032), fulvestrant and letrozole (NCT02684032), and cisplatin and docetaxel (NCT01920061) (ClinicalTrials.gov, accessed on 7 December 2020). Preliminary results from a recent ongoing trial showed that gedatolisib exhibited antitumor activity and a tolerable safety profile when combined with carboplatin and weekly PTX in the treatment of advanced solid tumors (NCT02069158) [19]. New clinical trials are needed to test the combination of gedatolisib, PTX, and ICI therapies in BC. Given the preferentially high expression levels of PI3KCD and PIK3CG in TNBC, and the preclinical data from this study, along with the data from the existing clinical trials testing gedatolisib + PTX, we suggest that combined treatment with gedatolisib, PTX, and ICI will lead to a durable therapeutic response for TNBC patients.

\section{Methods}

\subsection{Patients and Samples}

This study was conducted according to the Vanderbilt University Medical Center IRB, approval number 140264 (13 November 2015-13 November 2018). All patient donors signed an informed consent before providing tissue samples. Human granulocytes were isolated by gradient centrifugation from fresh peripheral blood of 8 breast cancer patients who were $\mathrm{HR}^{+} / \mathrm{HER}^{+}{ }^{+}, \mathrm{HR}^{+} / \mathrm{HER}^{-}{ }^{-}$, or $\mathrm{HR}^{-} / \mathrm{HER} 2^{-}$enrolled in clinical trials NCT01791478, NCT01872260, or NCT02379247 at Vanderbilt University Medical Center. Detailed trial design and clinical characteristics of the patients enrolled were published previously [15]. Briefly, patients enrolled in NCT01791478 and NCT01872260 Arm 2 were given 250-600 mg (escalating dose) of alpelisib daily for 28 days, and $2.5 \mathrm{mg}$ of letrozole daily for 28 days. Patients enrolled in NCT02379247 were given 250-600 mg (escalating dose) of alpelisib daily and $100 \mathrm{mg} / \mathrm{kg}$ of nab-paclitaxel on days 1,8, and 15 of each 28-day cycle. The granulocyte layer at the interface of Histopaque(H)-1077 (Sigma, St. Louis, MO, USA) and H-1119 (Sigma) were collected by density gradient centrifugation [33]. Efforts were made to collect blood at each cycle of therapy, though in some cases it was not possible to obtain blood at every cycle. For killing assays, 30-fold peripheral blood-sorted granulocytes were co-cultured for $18 \mathrm{~h}$ with luciferase reporter-bearing MCF7 or MDA-MB-231 human breast cancer cells (obtained from ATCC, Manassas, VA, USA). Plate-attached tumor cells were lysed in situ and luciferase activity was recorded with GloMax luminescent reader (Promega, Madison, WI, USA).

\subsection{Mouse Studies}

The polyomavirus middle T (PyMT) breast cancer cell line was provided by the Hal Moses laboratory (Vanderbilt, Nashville, TN, USA) and had been previously characterized in our lab [34]. Cells were maintained in Dulbecco's Modification of Eagle's Medium (DMEM)-F12 medium in a humidified $5 \% \mathrm{CO}_{2}$ incubator at $37^{\circ} \mathrm{C}$. Cell media was supplemented with $10 \%$ heat-inactivated fetal bovine serum (FBS) and $1 \%$ penicillin-streptomycin 
(P/S) solution, and cultures were tested monthly for mycoplasma (e-MycoTM Plus, LiliF Diagnostics, Gyeonggi-do, Korea). PyMT cells $\left(1 \times 10^{5}\right)$ were injected into the fourth mammary fat pad of 8-10-week-old C57BL/6 mice. Mice were sorted to have similar average starting volume among treatments. Mouse body weight was assessed once a week and tumor measurements were taken twice a week with micro-calipers. Tumor volume was estimated as $0.5 \times$ length $\times$ width $\times$ width. Treatment began when tumors reached $50-150 \mathrm{~mm}^{3}$ volume on average or 1 week post-tumor cell implantation and continued until tumors exceeded $15 \mathrm{~mm}$ in diameter or became perforated. Drug dosage and delivery of all drugs are summarized in Supplementary Table S1. Animal studies were approved by the Vanderbilt IACUC under protocol M1600058-01 (17 July 2016-17 July 2019) and M1900098-00 (28 October 2019 to 28 October 2022) and conducted in accordance with IACUC guidelines.

\subsection{Multicolor Flow Cytometry}

To phenotype immune cells in the blood, bone marrow, and tumor, approximately $10^{6}$ cells were isolated, stained with LIVE/DEAD ${ }^{\mathrm{TM}}$ Fixable Aqua Dead Cell Stain Kit (ThermoFisher, Grand Island, NY, USA), and blocked using an anti-mouse CD16/CD32 antibody (BD Biosciences, San Jose, CA, USA) for $20 \mathrm{~min}\left(4^{\circ} \mathrm{C}\right)$ and then labeled with monoclonal antibodies (Biolegend, San Diego, CA USA) against markers of interest. The antibodies used in the mouse study include: CD45-APC/Cy7, Ly6G-PE/Cy7, CD11c-BV421, F4/80APC, CD11b-Alexa488, Ly6C-BV605, MHCII-AlexaFlour700, CD206-PE, CD8-PerCP/Cy5.5, CD103-BV785, CD3-PerCP/eFluor710, CD4-BV421, CD8-AlexaFlour700, NK1.1-FITC, PDL1-PE, CD69-APC, CD25-PE/Cy7, TIM3-BV605, LAG3-BV785, CD44-BV650, and CD62LBV570. Cell were washed and resuspended in fixation buffer (PBS containing $1 \%$ formalin). A total of up to 200,000 single cell events were collected using a 4-Laser Fortessa and the data were analyzed using FlowJo 10.5.3 software.

\subsection{Analysis of Publicly Available Dataset}

Single cell RNA-seq results of primary breast cancer cells and lymph node metastases from 11 patients representing the four subtypes of breast cancer (luminal A, luminal B, HER2, and triple-negative breast cancer) were obtained from Single Cell Expression Atlas (https:/ / www.ebi.ac.uk/gxa/sc/experiments/E-GEOD-75688/) (accessed on 7 December 2020). [35]. A total of 539 cells were sequenced from individual tumor tissues containing carcinoma and non-carcinoma microenvironment cells. Further t-distributed stochastic neighbor embedding (t-SNE) analyses were used to visualize the results. For all $\mathrm{t}$-SNE plots, perplexity $=25$, color plot was generated by cluster number $\mathrm{K}=10$.

\subsection{Statistical Analysis}

Statistical analyses were performed using R 3.6.0 or GraphPad Prism software version 8.3.0. Data are summarized in figures expressed as mean \pm SD. Treatment effects in standard two-group experiments were compared using a two-sample t-test with unequal variances or the Wilcoxon rank-sum test. For more than two-group experiments, a oneway ANOVA with post hoc test was used to compare the differences between treatments. For clinical trial analysis, fold-change was calculated for the change in $\mathrm{CD} 45^{+} \mathrm{CD} 6 \mathrm{~b}^{+}$ granulocyte population in the peripheral blood from the $8 \mathrm{BC}$ patients with complete cycle 1 data. In addition, the duration of response was calculated for each of the 8 patients, and a correlation analysis was performed on the fold-change of $\mathrm{CD} 45^{+} \mathrm{CD} 66 \mathrm{~b}^{+}$granulocyte population in relation to the duration of patient response. The Spearman correlation coefficient or a quadratic polynomial regression was used to evaluate the association between two continuous variables. Where indicated, ${ }^{*} p<0.05 ;{ }^{* *} p<0.01$; ${ }^{* * *} p<0.001$; and ${ }^{* * * *} p<0.0001$.

Supplementary Materials: The following are available online at https://www.mdpi.com/article/10 $.3390 /$ ijms22105207/s1, Figure S1: Immune profiling of tumors in mice treated with PI3K $\alpha$ isoform specific inhibitor, alpelisib, paclitaxel and immunotherapy, Figure S2: Alpelisib induces beneficial, 
but not durable, granulocyte responses in breast cancer patients, Figure S3: Gedatolisib + Immune induces systemic responses of DC and CD8+ T cells, Table S1: Drug and dosing information.

Author Contributions: C.Y., J.Y., and A.R. designed the research approach; C.Y., J.Y., and N.S. performed the experiments; C.Y. and J.Y. analyzed data; S.-C.C., G.D.A., C.Y., and J.Y. performed statistical analyses; V.G.A. and I.A.M. provided access to key patient tissue samples. C.Y. made a draft of the paper; C.Y. and A.R. revised and edited the paper. All authors have read and agreed to the published version of the manuscript.

Funding: This research was funded by the following grants: R01 CA34590(AR), R01 CA243326(AR), VA SRCS Award IK6BX005225 (AR), VA MERIT Award 101BX002301 (AR) and P30 CA068485.

Institutional Review Board Statement: The clinical trial study was conducted according to the Vanderbilt University Medical Center IRB, approval number 140264 (13 November 2015-13 November 2018). Animal studies were approved by the Vanderbilt IACUC under protocol M1600058-01 (17 July 2016-17 July 2019) and M1900098-00 (28 October 2019 to 28 October 2022) and conducted in accordance with IACUC guidelines.

Informed Consent Statement: Informed consent was obtained from all subjects involved in the study.

Data Availability Statement: Publicly available datasets were analyzed in this study. This data can be found here: Single Cell Expression Atlas (https://www.ebi.ac.uk/gxa/sc/experiments/E-GEOD75688/) (accessed on 7 December 2020).

Acknowledgments: We are indebted to Kerry W. Vazquez for facilitating these studies. We thank Stacey Mont, Rebecca L. Shattuck-Brandt, Anna E. Vilgelm, and Carly B. Williams for their insights and helpful discussion while this work was ongoing.

Conflicts of Interest: V.G.A.: Consulting/advisory roles for Daiichi Sankyo, Eisai and Novartis; Research funding from Astellas Pharma, Genentech/Roche, and Lilly. I.A.M: Institutional research funding from Pfizer, Genentech, Astra-Zeneca, Immunomedics, Lilly, Novartis, Puma; Ad board consulting for Pfizer, Genentech, Astra-Zeneca, Immunomedics, Lilly, Novartis, Macrogenics, SeaGen, Abbvie, Puma, GSK, Cyclacel, Blueprint. All other coauthors have declared that no additional conflict of interest exists.

\section{References}

1. Ellis, M.J.; Perou, C.M. The genomic landscape of breast cancer as a therapeutic roadmap. Cancer Discov. 2013, 3, 27-34. [CrossRef] [PubMed]

2. Vanhaesebroeck, B.; Guillermet-Guibert, J.; Graupera, M.; Bilanges, B. The emerging mechanisms of isoform-specific PI3K signalling. Nat. Rev. Mol. Cell Biol. 2010, 11, 329-341. [CrossRef] [PubMed]

3. Costa, R.L.B.; Han, H.S.; Gradishar, W.J. Targeting the PI3K/AKT/mTOR pathway in triple-negative breast cancer: A review. Breast Cancer Res. Treat 2018, 169, 397-406. [CrossRef]

4. Topalian, S.L.; Drake, C.G.; Pardoll, D.M. Immune checkpoint blockade: A common denominator approach to cancer therapy. Cancer Cell 2015, 27, 450-461. [CrossRef]

5. Gordon, S.R.; Maute, R.L.; Dulken, B.W.; Hutter, G.; George, B.M.; McCracken, M.N.; Gupta, R.; Tsai, J.M.; Sinha, R.; Corey, D.; et al. PD-1 expression by tumour-associated macrophages inhibits phagocytosis and tumour immunity. Nature 2017, 545, 495-499. [CrossRef]

6. Bates, J.P.; Derakhshandeh, R.; Jones, L.; Webb, T.J. Mechanisms of immune evasion in breast cancer. BMC Cancer 2018, 18, 556. [CrossRef]

7. Pfirschke, C.; Engblom, C.; Rickelt, S.; Cortez-Retamozo, V.; Garris, C.; Pucci, F.; Yamazaki, T.; Poirier-Colame, V.; Newton, A.; Redouane, Y.; et al. Immunogenic Chemotherapy Sensitizes Tumors to Checkpoint Blockade Therapy. Immunity 2016, 44, 343-354. [CrossRef]

8. Schmid, P.; Rugo, H.S.; Adams, S.; Schneeweiss, A.; Barrios, C.H.; Iwata, H.; Dieras, V.; Henschel, V.; Molinero, L.; Chui, S.Y.; et al. Atezolizumab plus nab-paclitaxel as first-line treatment for unresectable, locally advanced or metastatic triple-negative breast cancer (IMpassion130): Updated efficacy results from a randomised, double-blind, placebo-controlled, phase 3 trial. Lancet Oncol. 2020, 21, 44-59. [CrossRef]

9. Morgillo, F.; Della Corte, C.M.; Diana, A.; Mauro, C.D.; Ciaramella, V.; Barra, G.; Belli, V.; Franzese, E.; Bianco, R.; Maiello, E.; et al. Phosphatidylinositol 3-kinase (PI3Kalpha)/AKT axis blockade with taselisib or ipatasertib enhances the efficacy of anti-microtubule drugs in human breast cancer cells. Oncotarget 2017, 8, 76479-76491. [CrossRef] 
10. Sai, J.; Owens, P.; Novitskiy, S.V.; Hawkins, O.E.; Vilgelm, A.E.; Yang, J.; Sobolik, T.; Lavender, N.; Johnson, A.C.; McClain, C.; et al. PI3K inhibition reduces mammary tumor growth and facilitates antitumor immunity and anti-PD1 responses. Clin. Cancer Res. 2017, 23, 3371-3384. [CrossRef]

11. Benefit mixed with caution for buparlisib. Cancer Discov. 2017, 7, 121. [CrossRef]

12. Jovanovic, B.; Mayer, I.A.; Mayer, E.L.; Abramson, V.G.; Bardia, A.; Sanders, M.E.; Kuba, M.G.; Estrada, M.V.; Beeler, J.S.; Shaver, T.M.; et al. A randomized phase II neoadjuvant study of cisplatin, paclitaxel with or without everolimus in patients with stage II/III triple-negative breast cancer (TNBC): Responses and long-term outcome correlated with increased frequency of DNA damage response gene mutations, TNBC subtype, AR status, and Ki67. Clin. Cancer Res. 2017, 23, 4035-4045. [CrossRef] [PubMed]

13. Juric, D.; Janku, F.; Rodon, J.; Burris, H.A.; Mayer, I.A.; Schuler, M.; Seggewiss-Bernhardt, R.; Gil-Martin, M.; Middleton, M.R.; Baselga, J.; et al. Alpelisib plus fulvestrant in PIK3CA-altered and PIK3CA-wild-type estrogen receptor-positive advanced breast cancer: A phase 1b clinical trial. JAMA Oncol. 2019, 5, e184475. [CrossRef] [PubMed]

14. Teo, Z.L.; Versaci, S.; Dushyanthen, S.; Caramia, F.; Savas, P.; Mintoff, C.P.; Zethoven, M.; Virassamy, B.; Luen, S.J.; McArthur, G.A.; et al. Combined CDK4/6 and PI3Kalpha inhibition is synergistic and immunogenic in triple-negative breast cancer. Cancer Res. 2017, 77, 6340-6352. [CrossRef] [PubMed]

15. Williams, C.B.; Nebhan, C.A.; Yang, J.; Starnes, L.S.; Yan, C.; Vilgelm, A.E.; Chen, S.C.; Dan Ayers, G.; Abramson, V.; Mayer, I.A.; et al. Correlative studies investigating effects of PI3K inhibition on peripheral leukocytes in metastatic breast cancer: Potential implications for immunotherapy. Breast Cancer Res. Treat 2020, 184, 357-364. [CrossRef] [PubMed]

16. De Henau, O.; Rausch, M.; Winkler, D.; Campesato, L.F.; Liu, C.; Cymerman, D.H.; Budhu, S.; Ghosh, A.; Pink, M.; Tchaicha, J.; et al. Overcoming resistance to checkpoint blockade therapy by targeting PI3Kgamma in myeloid cells. Nature 2016, 539, 443-447. [CrossRef]

17. Magagnoli, M.; Carlo-Stella, C.; Santoro, A. Copanlisib for the treatment of adults with relapsed follicular lymphoma. Expert Rev. Clin. Pharmacol. 2020, 13, 813-823. [CrossRef] [PubMed]

18. Mallon, R.; Feldberg, L.R.; Lucas, J.; Chaudhary, I.; Dehnhardt, C.; Santos, E.D.; Chen, Z.; dos Santos, O.; Ayral-Kaloustian, S.; Venkatesan, A.; et al. Antitumor efficacy of PKI-587, a highly potent dual PI3K/mTOR kinase inhibitor. Clin. Cancer Res. 2011, 17, 3193-3203. [CrossRef]

19. Colombo, I.; Genta, S.; Martorana, F.; Guidi, M.; Samartzis, E.S.P.; Brandt, S.; Gaggetta, S.; Moser, L.; Pascale, M.R.; Terrot, T.; et al. Phase I dose-escalation study of the dual PI3K/mTORC1/2 inhibitor Gedatolisib (PF-05212384) in combination with paclitaxel (P) and carboplatin (C) in patients (pts) with advanced solid tumours. Ann. Oncol. 2020, 31, S487. [CrossRef]

20. Perou, C.M.; Sorlie, T.; Eisen, M.B.; van de Rijn, M.; Jeffrey, S.S.; Rees, C.A.; Pollack, J.R.; Ross, D.T.; Johnsen, H.; Akslen, L.A.; et al. Molecular portraits of human breast tumours. Nature 2000, 406, 747-752. [CrossRef]

21. Sorlie, T.; Perou, C.M.; Tibshirani, R.; Aas, T.; Geisler, S.; Johnsen, H.; Hastie, T.; Eisen, M.B.; van de Rijn, M.; Jeffrey, S.S.; et al. Gene expression patterns of breast carcinomas distinguish tumor subclasses with clinical implications. Proc. Natl. Acad. Sci. USA 2001, 98, 10869-10874. [CrossRef]

22. Duraiswamy, J.; Ibegbu, C.C.; Masopust, D.; Miller, J.D.; Araki, K.; Doho, G.H.; Tata, P.; Gupta, S.; Zilliox, M.J.; Nakaya, H.I.; et al. Phenotype, function, and gene expression profiles of programmed death-1(hi) CD8 T cells in healthy human adults. J. Immunol. 2011, 186, 4200-4212. [CrossRef]

23. Fridlender, Z.G.; Sun, J.; Kim, S.; Kapoor, V.; Cheng, G.; Ling, L.; Worthen, G.S.; Albelda, S.M. Polarization of tumor-associated neutrophil phenotype by TGF-beta: “N1" versus “N2" TAN. Cancer Cell 2009, 16, 183-194. [CrossRef]

24. Nicolas-Avila, J.A.; Adrover, J.M.; Hidalgo, A. Neutrophils in homeostasis, immunity, and cancer. Immunity 2017, 46, 15-28. [CrossRef]

25. Mantovani, A.; Cassatella, M.A.; Costantini, C.; Jaillon, S. Neutrophils in the activation and regulation of innate and adaptive immunity. Nat. Rev. Immunol. 2011, 11, 519-531. [CrossRef] [PubMed]

26. Ali, K.; Soond, D.R.; Pineiro, R.; Hagemann, T.; Pearce, W.; Lim, E.L.; Bouabe, H.; Scudamore, C.L.; Hancox, T.; Maecker, H.; et al. Inactivation of PI(3)K p110delta breaks regulatory T-cell-mediated immune tolerance to cancer. Nature 2014, 510, 407-411. [CrossRef]

27. Schmid, M.C.; Avraamides, C.J.; Dippold, H.C.; Franco, I.; Foubert, P.; Ellies, L.G.; Acevedo, L.M.; Manglicmot, J.R.; Song, X.; Wrasidlo, W.; et al. Receptor tyrosine kinases and TLR/IL1Rs unexpectedly activate myeloid cell PI3kgamma, a single convergent point promoting tumor inflammation and progression. Cancer Cell 2011, 19, 715-727. [CrossRef]

28. Carnevalli, L.S.; Sinclair, C.; Taylor, M.A.; Gutierrez, P.M.; Langdon, S.; Coenen-Stass, A.M.L.; Mooney, L.; Hughes, A.; Jarvis, L.; Staniszewska, A.; et al. PI3Kalpha/delta inhibition promotes anti-tumor immunity through direct enhancement of effector CD8(+) T-cell activity. J. Immunother. Cancer 2018, 6, 158. [CrossRef]

29. Pardoll, D.M. The blockade of immune checkpoints in cancer immunotherapy. Nat. Rev. Cancer 2012, 12, 252-264. [CrossRef] [PubMed]

30. Sharma, P.; Allison, J.P. The future of immune checkpoint therapy. Science 2015, 348, 56-61. [CrossRef]

31. Ooms, L.M.; Binge, L.C.; Davies, E.M.; Rahman, P.; Conway, J.R.; Gurung, R.; Ferguson, D.T.; Papa, A.; Fedele, C.G.; Vieusseux, J.L.; et al. The inositol polyphosphate 5-phosphatase PIPP regulates AKT1-dependent breast cancer growth and metastasis. Cancer Cell 2015, 28, 155-169. [CrossRef] [PubMed] 
32. Miles, D.W.; Gligorov, J.; André, F.; Cameron, D.; Schneeweiss, A.; Barrios, C.H.; Xu, B.; Wardley, A.M.; Kaen, D.; Andrade, L.; et al. LBA15 Primary results from IMpassion131, a double-blind placebo-controlled randomised phase III trial of first-line paclitaxel (PAC) \pm atezolizumab (atezo) for unresectable locally advanced/metastatic triple-negative breast cancer (mTNBC). Ann. Oncol. 2020, 31, S1147-S1148. [CrossRef]

33. Swamydas, M.; Lionakis, M.S. Isolation, purification and labeling of mouse bone marrow neutrophils for functional studies and adoptive transfer experiments. J. Vis. Exp. 2013, 10, e50586. [CrossRef]

34. Yang, J.; Yan, C.; Vilgelm, A.E.; Chen, S.C.; Ayers, G.D.; Johnson, C.A.; Richmond, A. Targeted deletion of CXCR2 in myeloid cells alters the tumor immune environment to improve antitumor immunity. Cancer Immunol. Res. 2021, 9, 200-213. [CrossRef]

35. Chung, W.; Eum, H.H.; Lee, H.O.; Lee, K.M.; Lee, H.B.; Kim, K.T.; Ryu, H.S.; Kim, S.; Lee, J.E.; Park, Y.H.; et al. Single-cell RNA-seq enables comprehensive tumour and immune cell profiling in primary breast cancer. Nat. Commun. 2017, 8, 15081. [CrossRef] 